\title{
Experiential learning in community pharmacy: Online and remote teaching experience in Malaysian higher education
}

\author{
N.S.A Rahman*, N.I.M. Nazar, M.H. Elnaem \\ Department of Pharmacy Practice, Kulliyyah of Pharmacy, International Islamic University Malaysia, Malaysia
}

\author{
Keywords \\ Experiential Learning \\ Community Pharmacy \\ Malaysia \\ Online Teaching
}

${ }^{*}$ Corresponding author norny@iium.edu.my

\begin{abstract}
Summary: Experiential learning provides opportunities for students to learn beyond the classroom setting. Due to the COVID-19 pandemic, students were unable to complete their attachment modules in community pharmacy settings, thus a four week community pharmacy course was conducted via remote learning. Students were required to have a maximum of two hours of virtual discussion and consultation sessions with a faculty member (with community pharmacy background/experience) twice a week. In each week, students were required to complete all tasks given based on the activities proposed in the community pharmacy logbook. MyDispense (an online pharmacy simulation) and Access Pharmacy (McGraw-Hill) were utilised by students to practice their dispensing skills and familiarisation with non-prescription medications, respectively. Case discussions on responding to minor ailments were also touched on during the online sessions. Students were assessed via logbook, preceptor assessment and an online objective structured clinical examination (OSCE).
\end{abstract}

\begin{abstract}
Background and Context
Experiential learning can enhance the student-learning experience and prepare learners for success beyond the classroom (Miller, \& Olesova, 2015). Experiential learning in community pharmacy is just as important as in a hospital pharmacy or clinical setting. This method of learning serves as an important delivery method to expose and provide onjob training to undergraduate pharmacy students. By having exposure in a real setting, students are usually able to easily understand and grasp the knowledge to further apply it in the same setting. The implementation of experiential learning in community setting, however, was made difficult during the COVID-19 pandemic; this put the educators and students in a difficult situation. Pandemics should not deter the delivery of teaching and assessment. Therefore, the course required some innovations to address all the challenges faced during the pandemic.
\end{abstract}

\section{Educational Description}

The course was originally designed for students to do their attachments in community pharmacies for a four-week period. The delivery method is a form of experiential learning where, for the whole four weeks, teaching and learning is conducted in a community pharmacy setting. Due to the COVID-19 pandemic, where it was impossible for students to get involved in any fieldwork, the course had to be redesigned. Instead of an on-site attachment, it was replaced with synchronous discussions and consultation sessions using Google Meet and Google Classroom platforms. The course was shortened from four weeks to three weeks per rotation. In total there were 113 students registered for the course, in which 37 students were grouped in three different rotations.

The virtual sessions were conducted twice a week whereby, in each session students were required to prepare some tasks assigned to them beforehand. The topics discussed 
included community pharmacy set up, products or drug procurement procedures, prescription screening, dispensing, customer services, counselling, selling products, drugs/ product consultation, searching drug information, wholesaling (if any) and business-related work. The students were given opportunities to learn from a range of products which included over the counter (OTC), non-prescription medicines (NPM), prescription medicines, psychotropics, dangerous drugs, healthcare products, skincare, cosmetics, rehabilitation products, wound care, etc. Apart from using the Google Meet and Google Classroom platforms, the department also used WhatsApp as a main communication tool. This was to keep the lecturers in the communication loop, and questions were also posted to students using the same communication tool. In order to expose the students to common activities in community pharmacy, which included medication dispensing and counselling, students were required to complete exercises on MyDispense system (MyDispense, 2020). They were also required to do online assessments on nonprescription medications (NPM) in Access Pharmacy (McGraw-Hill Medical, 2020). This was aimed to familiarise the students with NPM in a community pharmacy setting. As one of the course learning outcomes was for students to demonstrate competency in providing drug consultations in a community pharmacy setting, case discussions were also conducted during the virtual sessions.

A number of cases on minor ailments were shared with students a few days before the virtual sessions for preparation. Students were required to provide their responses to the cases given during the virtual sessions. Lecturers provided their comments on the students' responses and shared their experiences. There were three types of assessment: logbook, preceptor assessment, and an online objective structured clinical examination (OSCE). Students were required to submit the completed logbook by the end of the attachment. Meanwhile, the preceptor assessment was based on students' participation during the discussion sessions and the reports generated from the sessions conducted. Having an online OSCE also assessed students' competency and skills mainly in responding to the symptoms of the minor ailment. Students' feedback was obtained from the reflective diaries in their log book and using a Google Form survey. The survey was conducted at the end of the term, particularly after participation in the online OSCE.

\section{Outcomes and Recommendations}

The use of MyDispense was really helpful and eased the learning process as it used a virtual community pharmacy setting. It would, however, have been better to have a setting and medications that were relevant to students' specific community, culture, and setting, to avoid confusion among students. Cases made use of the MyDispense system the initial stage but the discussions had to be conducted with lecturers as MyDispense was unable to assess communication skills.

Two-way communication is way better than a one-way response from students. The authors conducted the virtual session with about 37 students and two more lecturers per session each rotation. It was challenging to handle a large group in an online discussion. Some students may not have actively participated in the discussions. The reason why discussions were conducted with such large groups was to ensure similar information was delivered to all students and to minimise variations.

\section{References}

McGraw-Hill Medical. (2020). Access Pharmacy. McGraw-Hill Medical. Available at: $\underline{\text { https://accesspharmacy.mhmedical.com }}$

Miller, D., \& Olesova, L., (2015). Designing and implementing experiential learning through multimedia-based activities and blogging. Available at: https://secure.onlinelearningconsortium.org/ effective_practices/designing-and-implementing-experientiallearning-through-multimedia-based-activi

MyDispense (2020). Virtual Practice Environment for Teaching Safe Dispensing. Available at: https://info.mydispense.monash.edu 